\title{
EARLY EXTUBATION AFTER CARDIAC OPERATIONS IN NEONATES AND YOUNG INFANTS
}

Jeffrey S. Heinle, MD

Laura K. Diaz, MD

Lawrence S. Fox, MD
Objective: This study was undertaken to determine the feasibility of early extubation of the neonate and young infant after surgical repair of congenital heart lesions. Methods: The records of all patients less than 90 days of age who had cardiac operations over a 1-year period were reviewed. During this time, all patients were managed as potential candidates for early extubation. Fifty-six patients are included with a mean age of $32 \pm 31$ days and a mean weight of $3.7 \pm 0.9 \mathrm{~kg}$. Results: Twenty-eight patients (50\%) were extubated in the operating room or within 3 hours after arriving in the intensive care unit. This included $38 \%$ of patients less than 7 days of age, $50 \%$ of patients 8 to 30 days of age, $44 \%$ of patients 31 to 60 days of age, and $69 \%$ of patients 61 to 90 days of age. Three patients $(11 \%)$ extubated early required reintubation. No deaths were related to early extubation. Only one patient was negatively affected by early extubation. Patients extubated early had shorter stays in the intensive care unit $(3.3 \pm 3.9$ vs $6.7 \pm 2.9$ days $)$ and shorter postoperative hospital stays ( $5.9 \pm 3.3$ vs $13.5 \pm 9.7$ days), as well as lower intensive care unit $\mathbf{\$ 5 , 8 5 1} \pm \$ \mathbf{\$}, 225$ vs $\$ 12,064 \pm \$ 4,419)$ and total hospital $(\$ 21,108 \pm \$ 14,941$ vs $\$ 31,608 \pm \$ 9,861)$ costs than patients who were ventilated. Conclusions: Early extubation can be accomplished safely in many neonates and young infants undergoing cardiac operations for repair of congenital heart defects and can shorten hospital stay and reduce costs. (J Thorac Cardiovasc Surg 1997;114:413-8)
$\mathrm{T}$ he postoperative management of children with congenital heart disease continues to evolve and differs from institution to institution. The stress response to cardiac surgical operations is greater in neonates and small infants than in older patients. ${ }^{1}$ The endotracheal tube, mechanical ventilation, and the interventions necessary to maintain them, especially suctioning, have been shown to be particularly stressful. $^{2}$ Thus many institutions advocate continued ventilation, high-dose narcotic sedation, and often paralysis during the initial postoperative recovery in an effort to minimize the stress response to the operation, prevent pulmonary hypertensive episodes, and improve outcome. ${ }^{2-5}$

Increasing interest and a trend toward earlier

From the Cook Children's Medical Center, Fort Worth, Tex.

Received for publication Feb. 27, 1997; revisions requested April 9, 1997; revisions received May 9, 1997; accepted for publication May 14, 1997.

Address for reprints: Lawrence S. Fox, MD, Director, Pediatric Cardiovascular Surgery, Cook Children's Medical Center, 801 Seventh Ave., Fort Worth, TX 76104.

Copyright (C) 1997 by Mosby-Year Book, Inc.

$0022-5223 / 97 \$ 5.00+0 \quad \mathbf{1 2 / 1 / 8 3 2 3 1}$ extubation in adults ${ }^{6-8}$ and children ${ }^{9-13}$ have shown that early extubation after cardiac operations is practical, may result in fewer pulmonary complications, shortens the stay in the intensive care unit (ICU) and hospital, is cost effective, and allows for better use of resources. The feasibility and safety of this approach for neonates and young infants is not as well documented. With continued improvement in the anesthetic, surgical, and perfusion management of patients with congenital heart disease, the need to continue ventilation and sedation into the postoperative period can be eliminated in many of these young patients. Furthermore, their perioperative courses can be planned in such a way that will allow many to be safely extubated at the completion of their operation.

This study reviews the experience at Cook Children's Medical Center with early extubation of the neonate and young infant after surgical repair of congenital heart lesions.

\section{Patients and methods}

The records of all patients 90 days of age or younger who had cardiac operations between November 1, 1995, and November 1, 1996, were reviewed. During this time 
Table I. Diagnosis

\begin{tabular}{lcc}
\multicolumn{1}{c}{ Diagnosis } & Study group & Previous year \\
\hline Single ventricle & 12 & 9 \\
TOF & 10 & 6 \\
TGA & 10 & 11 \\
Coarctation + other & 7 & 12 \\
VSD & 6 & 9 \\
TAPVC & 3 & 5 \\
AV canal & 3 & 1 \\
Other & 5 & 10 \\
Total & 56 & 63 \\
\hline
\end{tabular}

TOF, Tetralogy of Fallot; TGA, transposition of the greater arteries; VSD, ventricular septal defect; $T A P V C$, total anomalous pulmonary venous connection; $A V$ canal, atrioventricular septal defect.

period, all patients were managed as potential candidates for early extubation. Sixty-one patients had cardiac procedures during the study period. Five patients died and were excluded from analysis because they were never extubated and did not meet criteria for extubation. The remaining 56 patients comprise the study group; of this group, 21 were 7 days of age or younger at operation, 10 were 8 to 30 days, 9 were 31 to 60 days, and 16 were 61 to 90 days (mean $32 \pm 31$ days). The mean weight was $3.7 \pm$ $0.9 \mathrm{~kg}$. The diagnoses of these patients are shown in Table I as the study group.

Anesthetic management in this group consisted of mask induction of anesthesia with halothane if no intravenous access was present, midazolam hydrochloride $(0.1 \mathrm{mg} / \mathrm{kg})$ for amnesia, rocuronium bromide $(0.1 \mathrm{mg} / \mathrm{kg})$ for muscle relaxation initially and again before cardiopulmonary bypass (CPB), and a single dose of caudal morphine (Duramorph, 50 to $75 \mu \mathrm{g} / \mathrm{kg}$, diluted in normal saline solution, $1 \mathrm{ml} / \mathrm{kg}$ ) after endotracheal intubation. Anesthesia was maintained with isoflurane $(0.2 \%$ to $1 \%)$, titrated on the basis of clinical indicators of depth of anesthesia, and minimal narcotic (generally less than a total of 10 $\mu \mathrm{g} / \mathrm{kg}$ fentanyl). Additional doses of rocuronium were given as required for paralysis during the procedure. Neuromuscular blockade was reversed at the end of the operation with edrophonium $(1 \mathrm{mg} / \mathrm{kg})$ and atropine $(0.02$ $\mathrm{mg} / \mathrm{kg}$ ).

The CPB circuit consisted of a Bio-Medicus centrifugal arterial pump (Medtronic Bio-Medicus, Eden Prairie, Minn.) with a Terumo hollow-fiber membrane oxygenator (Terumo Corp., Tokyo, Japan). An asanguineous colloid solution was used to prime the system, with no effort made to adjust the hematocrit value before rewarming. The heart was manipulated as little as possible before the institution of CPB. A single dose of cold crystalloid cardioplegic solution was used for myocardial protection. Circulatory arrest was used in the majority of patients and, when used, an attempt was made to keep the cumulative arrest time less than 30 minutes. Low-flow hypothermic $\mathrm{CPB}(0.5 \mathrm{~L} / \mathrm{min}$ per square meter) was used when possible in an effort to minimize arrest times. Ultrafiltration was used throughout the CPB period. Fresh whole blood was administered during rewarming and at the completion of CPB.

After separation from $\mathrm{CPB}$, the patient's cardiac, pulmonary, and neurologic status were assessed to consider the feasibility of early extubation. In the absence of severe pulmonary dysfunction, cardiac instability, anatomic concerns about the airway, or severe bleeding, the patient was allowed to awaken and neuromuscular blockade was reversed. If the patient had an adequate ventilatory effort and satisfactory gas exchange, he or she was extubated. In this study early extubation is defined as extubation in the operating room or within 3 hours after arrival in the ICU. Study patients who were extubated within this time period form the early extubation group, and patients who were not extubated within this time period form the ventilated group. Patients who were not extubated early were generally placed on a regimen of continuous fentanyl (10 to 15 $\mu \mathrm{g} / \mathrm{kg}$ per hour) and often vecuronium $(0.1 \mathrm{mg} / \mathrm{kg}$ per hour) infusions.

In addition to this study group, the records of all patients 90 days of age or younger undergoing cardiac operations during the preceding year (November 1, 1994, through October 31, 1995) were also reviewed. During this time period, early extubation was not considered for these patients, and they were routinely sedated and often paralyzed in the postoperative period. Sixty-nine patients had cardiac operations during this period. Six patients died and were excluded from analysis because they were never extubated and did not meet the criteria for extubation. The remaining 63 patients comprise the previous year group. Twenty-two of these patients were 7 days of age or younger, 14 were 8 to 30 days, 17 patients were 31 to 60 days, and 10 patients were 61 to 90 days. The mean age was $28 \pm 28$ days and the mean weight was $3.4 \pm 0.9 \mathrm{~kg}$. The diagnoses for these patients are also listed in Table I.

Patient records were also reviewed for costs related to their hospitalization. These costs reflect direct patient costs and not hospital overhead or patient charges. Figures were calculated from the time of arrival in the ICU after the operation until the time of discharge from the ICU (ICU costs), time of arrival in the ICU after the operation until time of discharge from the hospital (postoperative costs), and from time of admission to the hospital until time of discharge from the hospital (total hospital costs). These latter costs include any preoperative and intraoperative costs.

Data are reported as the mean \pm standard deviation unless otherwise stated. Comparisons between the groups were analyzed by means of the unpaired Student's $t$ test or $\chi^{2}$ contingency tables. Statistical significance was accepted at the $95 \%$ confidence level $(p<0.05)$.

\section{Results}

Early extubation. Of the 56 patients comprising the study group, $28(50 \%)$ were able to be extubated in the operating room or within 3 hours after arriving in the ICU; these patients constitute the early extubation group. The remaining 28 patients were left intubated for an average of $3.4 \pm 2.7$ days; these patients comprise the ventilated group. Table II summarizes these groups by age at operation. Table III summarizes the outcome related to the 
Table II. Study group: Outcome by age at operation

\begin{tabular}{crrrrrr}
\hline & \multicolumn{3}{c}{$\begin{array}{c}\text { Early } \\
\text { extubation }\end{array}$} & & \multicolumn{2}{c}{ Ventilated } \\
\cline { 3 - 4 } Age & $n$ & No. & $\%$ & & No. & $\%$ \\
\hline 57 days & 21 & 8 & 38 & 13 & 62 \\
8-30 days & 10 & 5 & 50 & 5 & 50 \\
31-60 days & 9 & 4 & 44 & 5 & 56 \\
61-90 days & 16 & 11 & 69 & 5 & 31 \\
Total & 56 & 28 & 50 & 28 & 50
\end{tabular}

cardiac lesion. Only one patient in the previous year group was extubated early.

Reintubation. Of the 28 patients extubated early, three $(11 \%)$ required reintubation, for an overall successful early extubation rate of $45 \%$. Two of these patients were 7 days of age or less and one was 12 days old. The diagnosis was tricuspid atresia in two patients and interrupted aortic arch in the other patient. Two of these three patients were reintubated because of pulmonary dysfunction and one for postoperative bleeding necessitating reoperation. No important adverse effects from reintubation occurred in two of these reintubated patients. One patient was subsequently extubated 24 hours after reintubation and the other 32 hours after reintubation. One patient early in the experience, who had tricuspid atresia and had undergone atrial septectomy and placement of a modified Blalock-Taussig shunt, required reintubation and subsequent mechanical ventilation for 16 days and hospitalization for 44 days. He had multiple subsystem dysfunction as a result of the delayed recognition of the need for reintubation. Of the 28 patients in the ventilated group, two ( $7 \% ; p=0.64$ vs early extubation group) required reintubation after later extubation.

Deaths. Two deaths occurred in the early extubation group, but neither of these deaths was related to early extubation. One patient with tricuspid atresia died after reoperation for intracardiac thrombus, and one patient with pulmonary atresia and intact ventricular septum died on postoperative day 7 as a result of an apparent pulmonary hypertensive crisis. No deaths occurred in the ventilated group ( $p=$ 0.15 vs early extubation group).

Perioperative characteristics. Table IV summarizes the perioperative characteristics of the early extubation and ventilated groups. In addition, the lowest hematocrit value during CPB was $14 \% \pm 2 \%$ in the extubated group versus $15 \% \pm 3 \%$ in the ventilated group $(p=0.15)$. During CPB, $49 \% \pm$ $14 \%$ of the combined cardioplegic and pump prime
Table III. Study group: Outcome by lesion

\begin{tabular}{|c|c|c|c|c|c|}
\hline \multirow[b]{2}{*}{ Lesion } & \multirow[b]{2}{*}{$n$} & \multicolumn{2}{|c|}{$\begin{array}{c}\text { Early } \\
\text { extubation }\end{array}$} & \multicolumn{2}{|c|}{ Ventilated } \\
\hline & & No. & $\%$ & No. & $\%$ \\
\hline Single ventricle & 12 & 4 & 33 & 8 & 67 \\
\hline TOF & 10 & 7 & 70 & 3 & 30 \\
\hline TGA & 10 & 4 & 40 & 6 & 60 \\
\hline Coarctation + other & 7 & 3 & 43 & 4 & 57 \\
\hline VSD & 6 & 3 & 50 & 3 & 50 \\
\hline TAPVC & 3 & 2 & 67 & 1 & 33 \\
\hline AV canal & 3 & 1 & 33 & 2 & 67 \\
\hline Other & 5 & 4 & 80 & 1 & 20 \\
\hline Total & 56 & 28 & 50 & 28 & 50 \\
\hline
\end{tabular}

For legend see Table I.

volume was removed by ultrafiltration in the extubated group, and $49 \% \pm 10 \%$ was removed in the ventilated group $(p=0.90)$. Two patients $(7 \%)$ in each group had focal seizures in the postoperative period.

Patients who were not extubated early continued to have ventilatory support for an average of $3.4 \pm$ 2.7 days after the operation. Patients in the early extubation group had significantly shorter ICU stays $(3.3 \pm 3.9$ vs $6.7 \pm 2.9$ days, $p=0.0006)$ and postoperative hospital stays $(5.9 \pm 3.3$ vs $13.5 \pm 9.7$ days, $p=0.0004$ ) than patients in the ventilated group.

Previous year group. When the study group is compared with the previous year group, similarities can be noted in age $(32 \pm 31$ vs $28 \pm 28$ days, respectively, $p=0.44)$, American Society of Anesthesiologists (ASA) class ( $3.6 \pm 0.5$ vs $3.7 \pm 0.5, p=$ $0.09)$, and preoperative intubation (23\% vs $38 \%$, $p=0.08)$, catecholamine use $(13 \%$ vs $24 \%, p=$ 0.11 ), and prostaglandin infusion ( $45 \%$ vs $38 \%, p=$ $0.47)$. The percentage of patients requiring inotropic support after the operation was also similar (31\% vs $40 \%, p=0.32$ ). The previous year group was intubated for an average of $4.0 \pm 4.7$ days after the operation versus $1.7 \pm 2.6$ days in the study group $(p=0.002)$. These patients spent $6.5 \pm 3.8$ days in the ICU and $14.8 \pm 13.8$ days in the hospital after the operation versus $5.0 \pm 3.8$ days $(p=0.03)$ and $9.8 \pm 8.2$ days $(p=0.02)$, respectively, for patients in the study group.

Costs. Table V shows cost data for the early extubation, ventilated, and previous year groups. ICU and postoperative hospital costs were significantly less for the extubated group than for the ventilated group. 
Table IV. Perioperative characteristics

\begin{tabular}{|c|c|c|c|}
\hline & $\begin{array}{c}\text { Early } \\
\text { extubation }\end{array}$ & Ventilated & $\begin{array}{c}p \\
\text { Value }\end{array}$ \\
\hline Patients $(n)$ & 28 & 28 & \\
\hline Age (days) & $38 \pm 31$ & $27 \pm 30$ & 0.18 \\
\hline Weight $(\mathrm{kg})$ & $4.0 \pm 1.0$ & $3.5 \pm 0.8$ & 0.02 \\
\hline ASA class & $3.4 \pm 0.5$ & $3.8 \pm 0.5$ & 0.005 \\
\hline \multicolumn{4}{|l|}{ Preoperative: } \\
\hline Intubation $(n)$ & 5 & 8 & 0.34 \\
\hline Catecholamines $(n)$ & 2 & 5 & 0.23 \\
\hline Prostaglandins $(n)$ & 10 & 15 & 0.18 \\
\hline $\mathrm{CPB}$ time $(\mathrm{min})$ & $75 \pm 26$ & $96 \pm 31$ & 0.009 \\
\hline Ischemic time ( $\mathrm{min})$ & $42 \pm 23$ & $53 \pm 25$ & 0.09 \\
\hline $\mathrm{TCA}^{*}$ time $(\mathrm{min})$ & $26 \pm 11$ & $31 \pm 13$ & 0.18 \\
\hline \multicolumn{4}{|l|}{ Postoperative } \\
\hline Hematocrit (\%) & $27 \pm 5$ & $29 \pm 4$ & 0.17 \\
\hline $\mathrm{T}_{\text {bladder }}\left({ }^{\circ} \mathrm{C}\right)$ & $36 \pm 0.8$ & $36 \pm 0.9$ & 0.34 \\
\hline Inotropic support $(n)$ & 3 & 14 & 0.001 \\
\hline $\begin{array}{l}\text { Weight change } \\
\text { (\% of preop weight) }\end{array}$ & $2.6 \pm 4.5$ & $6.7 \pm 8.1$ & 0.02 \\
\hline \multicolumn{4}{|l|}{ Postextubation $\mathrm{ABG}$} \\
\hline $\mathrm{pH}$ & $7.398 \pm 0.043$ & $7.399 \pm 0.044$ & 0.98 \\
\hline $\mathrm{PaCO}_{2}(\mathrm{~mm} \mathrm{Hg})$ & $47 \pm 6$ & $38 \pm 5$ & $<0.0001$ \\
\hline Base express & $5.4 \pm 6.2$ & $-0.2 \pm 2.8$ & 0.001 \\
\hline
\end{tabular}

$C P B$, Cardiopulmonary bypass; $T C A$, total circulatory arrest, $T_{\text {bladden }}$ bladder temperature; $A B G$, arterial blood gas; $\mathrm{PaCO}_{2}$ arterial carbon dioxide tension.

*TCA was not used in six patients in the early extubation group and four patients in the ventilated group.

\section{Discussion}

The postoperative management of neonates and young infants after cardiac surgical procedures has received much attention in the literature as early corrective and palliative repairs have become the standard of care in these babies. Certainly the excellent outcome in this group of patients is due to a host of factors, including surgical technique, anesthetic management, and postoperative care. The use of high-dose narcotic anesthesia during operations for congenital heart disease and extending into the postoperative period has been recommended to blunt the stress response during surgery, provide for cardiovascular stability, avoid pulmonary vasoconstrictive responses and hemodynamic instability related to suctioning and stimulation, and improve mortality in this group of patients. ${ }^{2-5,14}$ Neonates have an accentuated stress response in the perioperative period, ${ }^{1}$ which is further amplified by manipulation of the endotracheal tube and endotracheal suctioning. ${ }^{2}$ This has led to the practice of continued intubation, ventilation, sedation, and often paralysis into the postoperative period. ${ }^{4,5}$

Other authors have reviewed the potential deleterious effects of mechanical ventilation including laryngotracheal trauma, mucus plugging, kinking of the endotracheal tube, accidental extubation, and infection. ${ }^{13-15}$ In addition, pulmonary hypertensive crises can be caused by manipulation of the endotracheal tube and by tracheal stimulation with suctioning. Furthermore, positive intrapulmonary pressure can increase pulmonary vascular resistance and may impede pulmonary blood flow. ${ }^{16}$ Earlier extubation may minimize many of these complications. ${ }^{11}$ Endotracheal extubation may actually improve cardiac performance by increasing stroke work and cardiac output in patients who have had cardiac operations. $^{17}$

Because of the potential problems with extended intubation, a policy of considering early extubation for all patients undergoing cardiac operations was adopted. This policy extends to neonates and young infants as well. This study reviews our experience with early extubation exclusively in neonates and infants less than 90 days of age. In this group of very young patients, nearly half were able to be successfully extubated early. Early extubation was safe in this group, with no deaths related to extubation and only one patient (early in the experience) having a complication related to early extubation. Patients operated on in the first week of life were less likely to be extubated early, and two of these patients required reintubation, for an overall successful early extubation rate in this group of $29 \%$. Two of the four patients with single ventricle who were extubated early required reintubation, for an overall early extubation rate of $17 \%$, and no patient undergoing a Norwood operation for hypoplastic left heart syndrome was extubated early. These data suggest that patients who are operated on in the first week of life or who have single ventricle physiology may not be appropriate candidates and should be given careful consideration before early extubation is attempted.

When the early extubation and ventilated groups are compared with respect to perioperative variables, the ventilated group had a higher ASA score, longer $\mathrm{CPB}$ times, more fluid retention, and more frequent need for postoperative inotropic support. These factors imply that this was a more complex group of patients, and therefore extubation may not be feasible as often in these patients as a group. This implication is consistent with that of other groups in which shorter CPB times and shorter durations of operation correlated with the ability to extubate patients in the early postoperative period..$^{9,11,13}$ In this study, the duration of CPB or aortic crossclamping or the use of circulatory arrest did not prevent extubation at the conclusion of the operation. Additionally, the preoperative use of inotropic agents 
Table V. ICU, postoperative, and total hospital costs*

\begin{tabular}{llll}
\hline & Early extubation & Ventilated & Previous year \\
\hline ICU & $\$ 5,851 \pm \$ 7,225$ & $\$ 12,064 \pm \$ 4,419 \dagger$ & $\$ 11,599 \pm \$ 7,451 \dagger$ \\
Postoperative & $\$ 7,379 \pm \$ 9,503$ & $\$ 15,277 \pm \$ 7,588 \dagger$ & $\$ 15,237 \pm \$ 12,499 \dagger$ \\
Total hospital & $\$ 21,108 \pm \$ 14,941$ & $\$ 31,608 \pm \$ 9,861 \dagger$ & $\$ 28,660 \pm \$ 18,284$ \\
\hline
\end{tabular}

*Average cost per patient.

$\dagger p \leq 0.005$ versus early extubation group.

or prostaglandins or the presence of preoperative intubation did not eliminate these patients from consideration for early extubation.

Patients in the early extubation group spent fewer postoperative days in the ICU and had shorter hospital stays, and this is reflected by lower ICU, postoperative hospital, and total hospital costs. Again, the length of ICU stay and hospitalization may reflect the complexity and severity of the patient's lesion and may not be directly related to the fact that the patient was able to be extubated early. Clearly, this is a limitation of a retrospective study such as this one. However, when compared with the study group, the previous year group was similar in age, presenting lesion, preoperative intubation, use of catecholamines and prostaglandins, and postoperative requirement for inotropic support. Yet, in the year before we instituted this early extubation policy, only one patient of 63 was extubated early whereas $50 \%$ of the patients during the study period were able to be extubated. Certainly this magnitude of difference is not due to patient factors alone but reflects a change in attitude, wherein all patients in the study group were considered to be potential candidates for early extubation. Patients who were extubated earlier left the ICU earlier and were able to be discharged earlier. If one accepts the premise that these two groups were similar, then potentially $50 \%$ of the previous year group could have been extubated early and spent an average of 3.4 fewer days in the ICU. This would translate into 107 fewer ICU days at an average ICU cost per day of $\$ 1824$ (calculated as total ICU costs divided by total ICU days) or a total potential ICU savings of $\$ 195,168$ for the year.

To be able to attempt early extubation at the end of the operation, we modified the techniques of anesthesia, perfusion, and surgery. Anesthesia was induced and maintained with the use of an inhalation technique with a minimal amount of narcotic (fentanyl $\leq$ $10 \mu \mathrm{g} / \mathrm{kg}$ ) administered during the operation. Clinically, this provided adequate anesthesia during the procedure while allowing early postoperative recovery and the potential for extubation in the operating room. Other authors have shown the safety of inhalational techniques in patients with congenital heart lesions, ${ }^{18,19}$ and the use of small doses of fentanyl to provide cardiac stability did not complicate extubation at the end of the procedure. ${ }^{12}$ Caudal anesthetics have been shown to provide excellent pain control and a decreased need for supplemental postoperative analgesics even in young infants ${ }^{20-22}$ and, although not proved in this study, may have contributed to earlier extubation by allowing for less intraoperative and postoperative narcotic administration.

The techniques of CPB and the use of ultrafiltration may also have contributed to the ability to extubate these patients early. A Bio-Medicus centrifugal arterial pump was used for CPB because of the reported reduced increase of hemolysis, ${ }^{23}$ microembolic activity, ${ }^{24}$ and complement activation ${ }^{25}$ when compared with roller pumps. Ultrafiltration was used during CPB to remove priming and cardioplegic volume and reduce concentrations of inflammatory mediators ${ }^{26}$ to avoid postoperative edema, and potentially improve pulmonary compliance and cardiac function. Postoperative weight gain was only $2.6 \%$ in those patients able to be extubated early. This is consistent with a review by DiCarlo and Steven, ${ }^{16}$ in which patients who were removed from mechanical ventilation were close to their preoperative weight whereas those in whom extubation failed had a significant increase in body weight.

Patients who are operated on in the first week of life and those with single ventricle physiology may not always be appropriate candidates for early extubation, and care should be taken when evaluating these patients for extubation. However, we apply the same perioperative management approach to these patients. In those patients who require prolonged ventilation, we continue sedation and often paralysis into the postoperative period.

The most important factor for early extubation may be to approach all of these patients as if they have the potential to be extubated early and then optimize the perioperative events to achieve this goal. When treated in this manner, neonates and young infants undergoing cardiac operations for 
repair of congenital heart defects can be successfully extubated early in the postoperative period. This leads to a more positive convalescence for the patient and family, shortens ICU and hospital stays, and reduces the cost of care.

We express our appreciation to Susan R. Staudt, MD, and Glenn R. Wyant, MD, for their contributions to anesthetic management; Roberto Caballero, MD, Larry E. Easterling, MD, and W. Britt Nelson, MD, our pediatric intensivists; Jonathan Womack, BS, CCP, and Jeane Gibson, RN, CCP, for their perfusion skills and help in data collection and analysis; and Carol Cronkrite for her help in manuscript preparation.

\section{REFERENCES}

1. Anand KJS, Hansen DD, Hickey PR. Hormonal-metabolic stress responses in neonates undergoing cardiac surgery. Anesthesiology 1990;73:661-70.

2. Hickey PR, Hansen DD, Wessel DL, Lang P, Jonas RA, Elixon EM. Blunting of stress responses in the pulmonary circulation of infants by fentanyl. Anesth Analg 1985;64:1137-42.

3. Jenkins J, Lynn A, Edmonds J, Barker G. Effects of mechanical ventilation on cardiopulmonary function in children after open-heart surgery. Crit Care Med 1985;13:77-80.

4. Hickey PR, Hansen DD. Pulmonary hypertension in infants: postoperative management. In: Yacoub M, editor. Annual of cardiac surgery: current science. 1989. p. 16-22.

5. Castaneda AR, Jonas RA, Mayer JE Jr, Hanley FL. Perioperative care. In: Cardiac surgery of the neonate and infant. Philadelphia: WB Saunders; 1994. p. 65-87.

6. Cheng DCH. Pro: Early extubation after cardiac surgery decreases intensive care unit stay and cost. J Cardiothorac Vasc Anesth 1995;9:460-4.

7. Higgins TL. Safety issues regarding early extubation after coronary artery bypass surgery. J Cardiothorac Vasc Anesth 1995;9:24-9.

8. Karski JM. Practical aspects of early extubation in cardiac surgery. J Cardiothorac Vasc Anesth 1995;9:30-3.

9. Burrows FA, Taylor RH, Hillier SC. Early extubation of the trachea after repair of secundum-type atrial septal defects in children. Can J Anaesth 1992;39:1041-4.

10. Neirotti RA, Alvarez CE, Campos MD, Quaglio MT, Vazquez JC. Total anomalous pulmonary venous connection. Scand J Thorac Cardiovasc Surg 1991;25:97-100.

11. Heard GG, Lamberti JJ, Park SM, Waldman JD, Waldman J. Early extubation after surgical repair of congenital heart disease. Crit Care Med 1985;13:830-2.

12. Schuller JL, Bovill JG, Nijveld A, Patrick MR, Marcelletti C.
Early extubation of the trachea after open heart surgery for congenital heart disease: a review of 3 years' experience. $\mathrm{Br} \mathrm{J}$ Anaesth 1984;56:1101-8.

13. Barash PG, Lescovich F, Katz JD, Talner NS, Stansel HCJ. Early extubation following pediatric cardiothoracic operation: a viable alternative. Ann Thorac Surg 1980;29:228-33.

14. Hansen DD, Hickey PR. Anesthesia for hypoplastic left heart syndrome: use of high dose fentanyl in 30 neonates. Anesth Analg 1986;65:127-32.

15. Koh SO, Bang SO, Hong YW, Cho HW, Cho BK. Incidence and predictors of postextubation laryngeal edema in pediatric patients with congenital heart disease. Yonsei Med J 1995;36: 53-7.

16. DiCarlo JV, Steven JM. Respiratory failure in congenital heart disease. Pediatr Clin North Am 1994;41:525-42.

17. Gall SA, Olsen CO, Reves JG, McIntyre RW, Tyson GS, Davis JW, et al. Beneficial effects of endotracheal extubation on ventricular performance: implications for early extubation after cardiac operations. J Thorac Cardiovasc Surg 1988;95:819-27.

18. Greeley WJ, Bushman GA, Davis DP, Reves JG. Comparative effects of halothane and ketamine on systemic arterial oxygen saturation in children with cyanotic heart disease. Anesthesiology 1986;65:666-8.

19. Laishley RS, Burrows FA, Lerman J, Roy WL. Effect of anesthetic induction regimens on oxygen saturation in cyanotic congenital heart disease. Anesthesiology 1986;65:673-7.

20. Krane EJ, Tyler DC, Jacobson LE. The dose response of caudal morphine in children. Anesthesiology 1989;71:48-52.

21. Rosen KR, Rosen DA. Caudal epidural morphine for control of pain following open heart surgery in children. Anesthesiology 1989;70:418-21.

22. Valley RD, Bailey AG. Caudal morphine for postoperative analgesia in infants and children: a report of 138 cases. Anesth Analg 1991;72:120-4.

23. Jakob HG, Hafner G, Thelemann C, Sturer A, Prellwitz W, Oelert H. Routine extracorporeal circulation with a centrifugal or roller pump. Trans ASAIO 1991;37:M487-9.

24. Trivedi UH, Turtle MRJ, Abboud S, Venn GE, Chambers DJ. Differences in microemboli production between roller pump and centrifugal pump during cardiopulmonary bypass: pathophysiology and techniques of cardiopulmonary bypass (abstract). 1996.

25. Wheeldon DR, Bethune DW, Gill RD. Vortex pumping for routine cardiac surgery: a comparative study. Perfusion 1990;5: $135-43$.

26. Wang MJ, Chiu IS, Hsu CM, Wang CM, Lin PL, Chang CI, et al. Efficacy of ultrafiltration in removing inflammatory mediators during pediatric cardiac operations. Ann Thorac Surg 1996;61:651-6. 